\title{
Exactly solvable reaction diffusion models on a Bethe Lattice through the empty-interval method
}

\author{
Laleh Farhang Matin
}

Received: 5 August 2014/ Accepted: 30 December 2014/Published online: 10 February 2015

(C) The Author(s) 2015. This article is published with open access at Springerlink.com

\begin{abstract}
The most general reaction-diffusion model on a Bethe Lattice with nearest-neighbor interactions is introduced, which can be solved exactly through the empty-interval method. The stationary solutions of such models are discussed. For some special choice of reaction rates the dynamics of the system is also studied.
\end{abstract}

Keywords Reaction-diffusion - Bethe Lattice .

Empty interval method

\section{Introduction}

Reaction-diffusion systems have been studied using various methods including analytical techniques, approximation methods, and simulation. Approximation methods are generally different in different dimensions, as for example the mean filed techniques, working good for high dimensions, generally do not give correct results for low-dimensional systems. A large fraction of analytical studies belong to low-dimensional (especially one-dimensional) systems, as solving low-dimensional systems should in principle be easier [1-11].

The Cayley tree or Bethe lattice is a tree (a lattice having no loops) where every site is connected to $\xi$ nearest neighbor sites. This no-loops property may allow exact solvability for some models, for general coordination number $\xi$ Reaction diffusion models on the Cayley tree are studied in, for example [12-17]. In [12, 13, 16] diffusionlimited aggregations, and in [14] two-particle annihilation

L. F. Matin ( $\square)$

Department of Physics, Islamic Azad University,

North Tehran Branch, Tehran, Iran

e-mail: laleh.matin@gmail.com reactions for immobile reactants have been studied. There are also some exact results for deposition processes on the Bethe lattice [17].

The empty interval method (EIM) has been used to analyze the one dimensional of diffusion-limited coalescence [18-21]. Using this method, the probability that $n$ consecutive sites are empty has been calculated. This method has been used to study a reaction-diffusion process with three-site interactions [22]. EIM has been also generalized to study the Kinetics of the q-state one-dimensional Potts model in the zero-temperature limit [23]. In [18-21] one-dimensional diffusion-limited processes have been studied using EIM. There, some of the reaction rates have been taken infinite, and the models have been worked out on continuum. For the cases of finite reaction-rates, some approximate solutions have been obtained.

In $[24,25]$, all the one-dimensional reaction-diffusion models with nearest neighbor interactions which can be exactly solved by EIM have been found and studied. Conditions have been obtained for the systems with finite reaction rates to be solvable via EIM, and then the equations of EIM have been solved. In [25], general conditions were obtained for a single-species reaction-diffusion system with nearest neighbor interactions, to be solvable through EIM. Here solvability means that evolution for $E_{n}$ (the probability that $n$ consecutive sites be empty) is closed. It turned out there, that certain relations between the reaction rates are needed, so that system is solvable via EIM. The evolution equation of $E_{n}$ is a recursive equation in terms of $n$, and is linear. It was shown that if certain reactions are absent, namely reactions that produce particles in two adjacent empty sites, the coefficients of the empty intervals in the evolution equation of the intervals are $n$-independent, so that the evolution equation can be easily solved. The criteria for solvability, and the solution 
of the empty-interval equation were generalized to cases of multi-species systems and multi-site interactions in [2628].

In this article, the most general single-species reactiondiffusion model with nearest-neighbor interactions on a Cayley tree is investigated, which can be solved exactly through the empty interval method. The scheme of the paper is as follows. In Sect. 2, the most general reactiondiffusion model with nearest neighbor interactions on a Cayley tree is studied, which can be solved exactly through EIM. The evolution equation of $E_{n}$, the probability that a connected collection of $\mathrm{n}$ sites be empty is also obtained. In Sect. 3 the stationary solutions of such models are discussed.

The dynamics of the system for special choice of reaction rates is studied in Sect. 4. Finally, Sect. 5 is devoted to concluding remarks.

\section{Models solvable through the empty interval method on a Cayley tree}

The Cayley tree (a lattice without loops) where every site is connected to $\xi$ sites (Figs. 1, 2). Two sites are called neighbors if they are connected through a link. Consider a system of particles on a Cayley tree. Each site is either empty or occupied by one particle. The interaction (of particles and vacancies) is nearest neighbor. The probability that a connected collection of $\mathrm{n}$ sites be empty is denoted by $E_{n}$. It is assumed that this quantity does not depend on the choice of the collection. An example is a tree where the probability that a site is occupied is $p$ and is independent of the states of other sites. Then

$E_{n}=(1-p)^{n}$.

The following graphical representations help express various relations in a more compact form. An empty

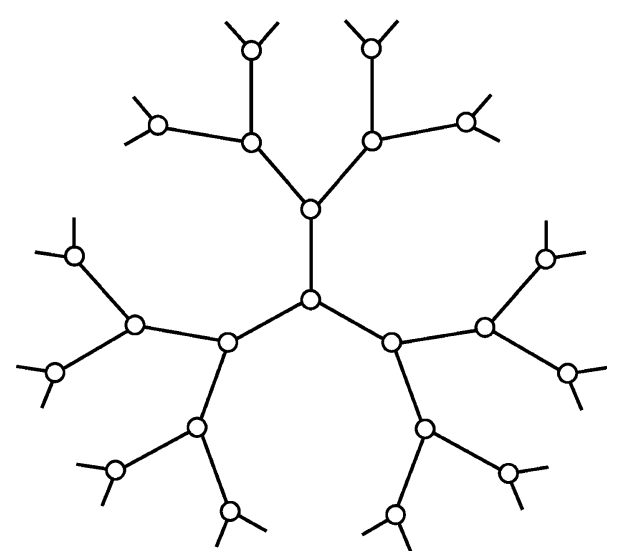

Fig. 1 The Cayley tree (Bethe lattice) with $\xi=3$

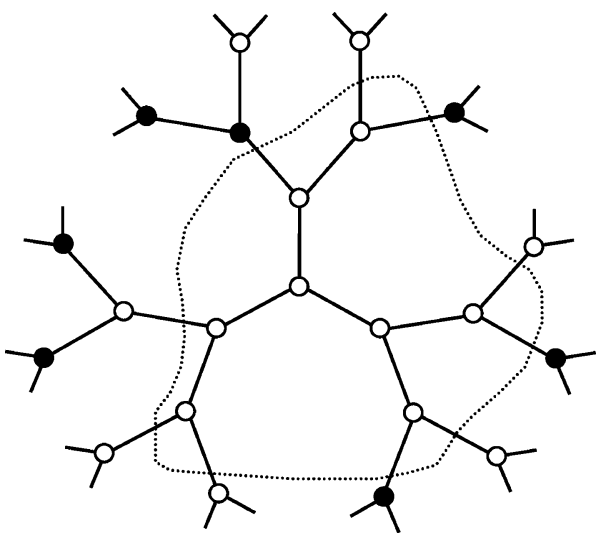

Fig. 2 An empty cluster with the links at the boundary, on a Cayley tree with $\xi=3$

(occupied) site is denoted by $o(\bullet)$. A connected collection of $n$ empty sites is denoted by $O_{n}$.

There is no loop in a Cayley tree, so each site can only be connected to a single existing cluster site, by a single link. An empty cluster expands or shrinks through interaction on its boundary. It can also be destroyed through particle generations in the bulk. Assume that the interactions are nearest neighbor (two-site) of the following kinds, with the rates indicated.

$\begin{aligned} \bullet \circ & \rightarrow\left\{\begin{array}{ll}\bullet \bullet, & r_{1} \\ \bullet & r_{2}\end{array}\right\}, \circ \rightarrow\left\{\begin{array}{ll}\bullet \circ, & r_{2} \\ \bullet \bullet, & r_{1}\end{array}\right\}, \bullet \\ & \rightarrow\left\{\begin{array}{ll}\bullet \circ, & r_{3} \\ \bullet \bullet, & r_{3}\end{array}\right\},\end{aligned}$

And

$\circ \circ\left\{\begin{array}{cc}\bullet \bullet, & r \\ \circ \bullet, & r_{0} \\ \bullet \circ, & r_{0}\end{array}\right\}$

The closeness of the evolution equation for $E_{n}$ requires that the rate of change of anything to (০o) becomes zero. The probability of finding an empty cluster connected to an occupied site $P\left(\bullet-O_{n}\right)$ can be written in terms of $E_{n}$ and $E_{n+1}$ :

$\begin{aligned} P\left(\bullet-O_{n}\right)+P\left(\circ-O_{n}\right) & =P\left(O_{n}\right) \Rightarrow P\left(\bullet-O_{n}\right) \\ & =E_{n}-E_{n+1}\end{aligned}$

To study the evolution of $E_{n}$, one considers states which can transform to $O_{n}$ (the sources) and states $O_{n}$ can transform to them (the sinks). These are the followings.

Sources: $\circ-\bullet-O_{n-1}, \bullet-\bullet-O_{n-1}$,
Sinks: $\bullet-O_{n}, \circ-O_{n}, O_{n}$.

Using these, the time evolution equation for $E_{1}(n>1)$ is seen to be 


$$
\begin{aligned}
\frac{\mathrm{d} E_{n}}{\mathrm{~d} t}= & R_{n}\left[r_{2} P\left(\circ-\bullet-O_{n-1}\right)+r_{3} P\left(\bullet-\bullet-O_{n-1}\right)\right] \\
& -R_{n}\left(r_{1}+r_{2}\right) P\left(\bullet-O_{n}\right)-R_{n}\left(r+r_{0}\right) P\left(\circ-O_{n}\right) \\
& -(n-1)\left(r+2 r_{0}\right) P\left(O_{n}\right),
\end{aligned}
$$

where $R_{n}$ is the number of sites adjacent to a collection of $n$ connected sites. A simple induction in [29] shows that

$R_{n}=n(\xi-2)+2$.

Using [4] and

$P\left(\circ-\bullet-O_{n-1}\right)+P\left(\bullet-\bullet-O_{n-1}\right)=P\left(\bullet-O_{n-1}\right)$,

equation [6] recasts to

$$
\begin{aligned}
\frac{\mathrm{d} E_{n}}{\mathrm{~d} t}= & R_{n}\left[r_{2}\left(E_{n-1}-E_{n}\right)+\left(r_{3}-r_{2}\right) P\left(\bullet-\bullet-O_{n-1}\right)\right. \\
& \left.-\left(r_{1}+r_{2}\right)\left(E_{n}-E_{n+1}\right)-\left(r+r_{0}\right) E_{n+1}\right] \\
& -(n-1)\left(r+2 r_{0}\right) E_{n}, \quad n>1 .
\end{aligned}
$$

In order that the evolution for $E_{n}$ be closed, it is needed that

$r_{3}=r_{2}$.

It is also seen that the evolution for $E_{1}$ takes a form similar to that of $E_{n}(n>1)$, provided one defines

$E_{0}=1$.

Then we have

$$
\begin{aligned}
\frac{\mathrm{d} E_{n}}{\mathrm{~d} t}= & R_{n}\left[r_{2}\left(E_{n-1}-E_{n}\right)-\left(r_{1}+r_{2}\right)\left(E_{n}-E_{n+1}\right)\right. \\
& \left.-\left(r+r_{0}\right) E_{n+1}\right]-(n-1)\left(r+2 r_{0}\right) E_{n} \quad n \geq 1 .
\end{aligned}
$$

along with the boundary condition [11].

\section{The stationary solution}

The stationary solution of the system $\left(E^{s}\right.$, for which the time derivative vanishes), satisfies

$$
\begin{aligned}
0= & R_{n} r_{2} E_{n-1}^{s}-\left[R_{n}\left(r_{1}+2 r_{2}\right)+(n-1)\left(r+2 r_{0}\right)\right] E_{n}^{s} \\
& +R_{n}\left(r_{1}+r_{2}-r-r_{0}\right) E_{n+1}^{s}, \quad n \geq 1,
\end{aligned}
$$

with the boundary condition

$E_{0}^{s}=1$.

Consider two cases separately.

$\boldsymbol{r}=\boldsymbol{r}_{0}=0$

One has

$r_{2} E_{n-1}^{s}-\left(r_{1}+2 r_{2}\right) E_{n}^{s}+\left(r_{1}+r_{2}\right) E_{n+1}^{s}=0$.
This is a second order linear difference equation with constant coefficients. To solve this, one puts the ansatz

$E_{n}^{s}=C_{1} z_{1}^{n}+C_{2} z_{2}^{n}$,

In [15], and obtains

$E_{n}^{s}=C_{1}+C_{2}\left(\frac{r_{2}}{r_{1}+r_{2}}\right)^{n}$.

The constants $C_{1}$ and $C_{2}$ depend on the initial conditions. So the stationary solution is not unique. Note that the result is independent of coordination number $\xi$. So the stationary behavior of the system, reaction diffusion model on a Cayley tree with arbitrary coordination number $\xi$, is similar to that of a reaction diffusion model on a onedimensional lattice $(\xi=2)$, provided of course that there is no process which creates particles from two neighboring vacant sites. Using [17] for $n=0$, and $n=1$, together with the boundary condition [14], the constants $C_{1}$ and $C_{2}$ can be expressed in term of $E_{1}^{s}$

$\boldsymbol{r} \neq 0$ or $\boldsymbol{r}_{0} \neq 0$

It is a difficult task to obtain a closed form for $E_{n}^{s}$. Things become, however, simpler for large $n$ 's. One puts the ansatz

$E_{n}^{s}=C_{n} z^{n}$,

In [13], assuming that $C_{n}$ is slowly varying with respect to $\mathrm{n}$. Keeping only the largest powers in $\mathrm{n}$, one arrives at $f(z)=0$.

where $f$ is defined through

$$
\begin{aligned}
f(z):= & (\xi-2)\left(r_{1}+r_{2}-r-r_{0}\right) z^{2} \\
& -\left[(\xi-2)\left(r_{1}+2 r_{2}\right)+\left(r+2 r_{0}\right)\right] z+(\xi-2) r_{2} .
\end{aligned}
$$

There are two solutions for $z$, each giving rise to a stationary solution. The general stationary solution would be linear combination of these. A stationary solution however, is acceptable only if it is nonnegative and nonincreasing for all $n$ 's. Such a solution should not blow up at $n \rightarrow \infty$, which shows that for acceptable solutions

$|z| \leq 1$.

Using

$f(0)=(\xi-2) r_{2}>0$,

$f(1)=-(\xi-2)\left(r+r_{0}\right)-\left(r+2 r_{0}\right)<0$,

It is seen that one of the roots of [20] (let's call it $z_{1}$ ) satisfies

$0<z_{1}<1$. 
For the other root, two cases occur:

(i) $r_{1}+r_{2}>r+r_{0}$.

Here $f(\infty)>0$, and $z_{2}>1$. Hence this root gives rise to $r_{0}$ an unphysical solution. So the stationary solution is unique.

(ii) $r_{1}+r_{2}<r+r_{0}$.

Here $f(\infty)<0$, and $z_{2}<0$. Using

$z_{1}+z_{2}=\frac{(\xi-2)\left(r_{1}+2 r_{2}\right)+r+2 r_{0}}{r_{1}+r_{2}-r-r_{0}}<0$,

One arrives at

$\left|z_{1}\right|<\left|z_{2}\right|$.

This shows that in any combination $C_{1 n} z_{1}^{n}+C_{2 n} z_{2}^{n}$, with $C_{1 n}$ and slowly varying, for large $n ' s$ the second term is the dominant term unless it is identical to zero. But the sign of this term changes with $n$ for large $n$. So the sign of the whole combination changes with $n$ for large $n$ 's unless the second term vanishes identically. Hence any combination of stationary solutions with a nonzero contribution corresponding to $z_{2}$ is unphysical. The stationary solution is again unique. Note that if one considers the evolution equation [12] on its own, without taking into account the fact that $E_{n} s$ are not totally independent of each other and cannot be negative, then there would be two stationary solutions. What eliminates one of these is that only special initial values are possible for $E_{n}$ ' $s$, so that in the large time limit only one of the stationary solutions survives.

To summarize, if $r$ or $r$ are nonvanishing, the stationary solution is unique, so the solution for large times is independent of the initial conditions.

There are two special subcases where [13] is simplified. In these cases [13], it is reduced to a first order difference equation.

$\boldsymbol{r}_{2}=0$

Here

$E_{n}^{s}=\delta_{n 0}$

is a solution to [13] and [14], and as the stationary solution is unique, this is the stationary solution.

$\boldsymbol{r}_{1}+\boldsymbol{r}_{2}=\boldsymbol{r}+\boldsymbol{r}_{0}$

In this case [13] becomes a first order recursive equation for $E_{n^{\prime}}^{s}$ from which on obtains

$E_{n}^{s}=\prod_{j=1}^{n} \frac{(\xi-2) r_{2} j+2 r_{2}}{\left[(\xi-2)\left(r_{1}+2 r_{2}\right)+\left(r+2 r_{0}\right)\right] j+2 r_{2}+r}$

\section{Dynamical solutions}

It is a difficult task to obtain the dynamical solution in general. One may seek solutions with exponential time dependence:

$E_{n}^{\varepsilon}(t)=E_{n}^{\varepsilon} \exp (\varepsilon t)$

Putting this in [12], one arrives at

$$
\begin{aligned}
0= & R_{n} r_{2} E_{n-1}^{\varepsilon}-\left[R_{n}\left(r_{1}+2 r_{2}\right)+(n-1)\left(r+2 r_{0}\right)+\varepsilon\right] E_{n}^{\varepsilon} \\
& +R_{n}\left(r_{1}+r_{2}-r-r_{0}\right) E_{n+1}^{\varepsilon}, \quad n \geq 1 .
\end{aligned}
$$

The boundary condition corresponding to [11] is

$E_{0}^{\varepsilon}=0$.

In general, it is difficult to solve these for large $n$, however, one can use arguments similar to those used in the previous section. Using an ansatz similar to [19], it is seen that $\varepsilon$ does not change the equation governing $z$. Again the root $z_{2}$ (which is either greater than one or negative) give rise to unphysical solutions. The reason is that although the dynamical solution [29] need not be nonnegative and decreasing in $n$ by itself, the sum of such a solution and the stationary solution can be a complete physical solution to [11] and [12] and hence should satisfy these conditions. For sufficiently large $n$, a contribution coming from $z_{2}$ would be dominant and either blows up or becomes negative. So the whole solution either blows up or becomes negative, unless there is no contribution corresponding to $z_{2}$ in the dynamical solutions. This leaves only one physical solution for (30). Such a solution does not necessarily satisfy (31). The condition (30) may be satisfied only for special values of $\varepsilon$. Hence the spectrum of the evolution operator is discrete, in other words the system has a finite relaxation time.

For two special choices of parameters, however, one can go further. These are the cases discussed in the previous section when the evolution equation becomes first order in $n$.

$\boldsymbol{r}_{2}=0$

Here the reactants are immobile, and the coagulation rate is also zero. But there are nonzero rates for birth and decoagulation. So, one expects at large times all the sites to be occupied, and in the stationary states all the $E_{n}^{\prime} s$ are zero as it was shown to be the case in the previous section. The evolution equation can be solved exactly for a special choice of initial condition, Eq. (12) recasts to

$$
\begin{aligned}
\frac{\mathrm{d} E_{n}}{\mathrm{~d} t}= & -\left(R_{n} r_{1}+(n-1)\left(r+2 r_{0}\right)\right] E_{n} \\
& +R_{n}\left(r_{1}-r-r_{0}\right) E_{n}+1, \quad n \geq 1 .
\end{aligned}
$$


Putting the ansatz

$E_{n}(t)=E_{1}(t)[b(t)]^{n-1}$,

In (32), one arrives at

$\frac{\mathrm{d} b}{\mathrm{~d} t}=-\left[(\xi-2)+r+2 r_{0}\right] b+(\xi-2)\left(r_{1}-r-r_{0}\right) b^{2}$,

$\frac{\mathrm{d} E_{1}}{\mathrm{~d} t}=\xi\left[-r_{1}+\left(r_{1}-r-r_{0}\right) b\right] E_{1}$.

These are readily solved and one obtains.

$b(t)=\frac{b(0) \exp (-\beta t)}{1+\alpha b(0)[1-\exp (-\beta t)],}$
$E_{1}(t)=E_{1}(0) \exp \left(-\xi r_{1} t\right)\left\{\frac{1}{1+\alpha b(0)[1-\exp (-\beta t)]}\right\}^{\frac{\xi}{\xi-2}}$,

where

$\alpha:=\frac{(\xi-2)\left(r+r_{0}-r_{1}\right)}{(\xi-2) r_{1}+r+2 r_{0}}$

$\beta:=(\xi-2) r_{1}+r+2 r^{\prime}$.

Using these, one obtains

$$
\begin{aligned}
E_{n}(t)= & E_{n}(0) \exp \left[-\xi r_{1} t-B(n-1) t\right] \\
& \times\left\{\frac{1}{1+\alpha b(0)[1-\exp (-\beta t)}\right\}^{\frac{\xi}{\xi-2}+n-1}
\end{aligned}
$$

It is seen that for times, all $E_{n} s$ tend to zero. In fact they decay like

$E_{n}(t) \sim \exp \left[-\xi r_{1} t-\beta(n-1) t\right]$.

A special case where the ansatz (33) works is the case of initially uncorrelated sites, so that each site is occupied with probability $\rho$ regardless of other sites.

One has then

$E_{n}(0)=(1-\rho)^{\wedge} n$,

So that

$E_{1}(0)=1-\rho$

$\boldsymbol{r}_{1}+\boldsymbol{r}_{2}=\boldsymbol{r}+\boldsymbol{r}_{0}$

In this case (12) becomes

$$
\begin{aligned}
\frac{\mathrm{d} E_{n}}{\mathrm{~d} t}= & R_{n} r_{2} E_{n-1}-\left[R_{n}\left(r_{1}+2 r_{2}\right)\right. \\
& \left.-(n-1)\left(r+2 r_{0}\right)\right] E_{n}, \quad n \geq 1
\end{aligned}
$$

This set of equations, together with the boundary condition [11], can be solved iteratively. The equation for $E_{1}(t)$ becomes $\frac{\mathrm{d} E_{1}}{\mathrm{~d} t}=\xi r_{2}-\xi\left(r_{1}+2 r_{2}\right) E_{1}$

the solution to which is

$E_{1}(t)=\frac{r_{2}}{r_{1}+2 r_{2}}+\left[E_{1}(0)-\frac{r_{2}}{r_{1}+2 r_{2}}\right] \exp \left[-\xi\left(r_{1}+2 r_{2}\right) t\right]$.

So, at large times each site is occupied whit the probability

$1-E_{n}(\infty)=\frac{r_{1}+r_{2}}{r_{1}+2 r_{2}}$

and the average density relaxes to this value with the relaxation time

$r=\frac{1}{\xi\left(r_{1}+2 r_{2}\right)}$

\section{Concluding remarks}

The most general one-dimensional single-species exclusion model on a Cayley tree was considered, for which the evolution of the empty-intervals is closed. The stationary solutions of such models were discussed. It was shown that except for special values of rates, the stationary solutions are unique, hence independent of initial conditions. The dynamics of such systems were also studied and it was shown that generally the spectrum is discrete and depends on $n$. For special choices of reaction rates, the dynamics was studied in more detail and closed form for the empty interval probabilities were obtained. Among the questions remaining one can mention the statics and dynamics of systems with arbitrary rates, as well as the possible existence of phase transitions. One can also investigate Cayley trees with boundaries, with injection and extraction at the boundaries.

Open Access This article is distributed under the terms of the Creative Commons Attribution License which permits any use, distribution, and reproduction in any medium, provided the original author(s) and the source are credited.

\section{References}

1. Schütz, G.M.: Exactly solvable models for many-body systems far from equilibrium. In: Domb, C., Lebowitz, J. (eds.) Phase Transitions and Critical Phenomena, vol. 19. Academic press, London (2000)

2. Alcaraz, F.C., Droz, M., Henkel, M., Rittenberg, V.: Reactiondiffusion processes, critical dynamics, and quantum chains. Ann. Phys. (N.Y.) 230, 250 (1994)

3. Krebs, K., Pfannmuller, M.P., Wehefritz, B., Hinrichsen, H.: Finite-size scaling studies of one-dimensional reaction-diffusion systems. J. Stat. Phys. 78(FS), 1429 (1995) 
4. Simon, H.: Concentration for one and two species one-dimensional reaction-diffusion. J. Phys. A28, 6585 (1995)

5. Privaman, V., Cadilhe, A.M.R., Glasser, M.L.: Exact solution of anisotropic diffusion limited reactions with coagulation and annihilation. J. Stat. Phys. 81, 881 (1995)

6. Henkel, M., Orlandini, E., Schütz, G.M.: Equivalences between stochastic systems. J. Phys. A28, 6335 (1995)

7. Henkel, M., Orlandini, E., Santos, J.: Reaction-diffusion processes from equivalent integrable quantum chains. Ann. Phys. 259, 163 (1997)

8. Lushnikov, A.A.: Binary reaction $1+1 \rightarrow 0$ in one dimension. Sov. Phys. JETP 64, 811 (1986). [Zh. Eksp.Teor.Fiz.91(1986)1376]

9. Alimohammadi, M., Karimipour, V., Khorrami, M.: Exact solution of a one-parameter family of asymmetric exclusion processes. Phys. Rev. E57, 6370 (1998)

10. Alimohammadi, M., Karimipour, V., Khorrami, M.: A twoparametric family of asymmetric exclusion processes and its exact solution. J. Stat. Phys. 97, 373 (1999)

11. Aghamohammadi, A., Khorrami, M.: Similarity transformation in one-dimensional reaction-diffusion systems: the voting model as an example. Phys. A33, 7843 (2000)

12. Vannimenus, J., Nickel, B., Hakim, V.: Models of cluster growth on the Cayley tree. Phys. Rev. B30, 391 (1984)

13. Krug, J.: Surface structure of random aggregates on the Cayley tree. J. Phys. A21, 4637 (1988)

14. Majumdar, S.N., Privman, V.: Annihilation of immobile reactions in the Bethe lattice. J. Phys. A26, L743 (1993)

15. Kelbert, M.Y., Suhov, Y.M.: The Markov branching random walk and systems of reaction-diffusion (Kolmogorov-PetrovskirPiskunov) equations. Commun. Math. Phys. 167, 607 (1995)

16. Majumdar, S.N.: Traveling front solutions to directed diffusion limited aggregation, digital search trees and the Lempel-Ziv data compression algorithm. Phys. Rev. E68, 026103 (2003)

17. Cadilhe, A., Privman, V.: Random sequential adsorption of mixtures of dimers and monomers on a pre-treated Bethe lattice. Modern Phys. Lett. B18, 207 (2004)
18. Burschka, M.A., Doering, C.R., Ben-Avraham, D.: Transition in the relaxation dynamics of a reversible diffusion-limited reaction. Phys. Rev. Lett. 63, 700 (1989)

19. Ben-Avraham, D.: The method of interparticle distribution functions for diffusion-reaction systems in one dimension. Phys. Lett. B9, 895 (1995)

20. Ben-Avraham, D.: Nonequilibrium statistical mechanics in one dimension. In: Privman, V. (ed), pp. 29-50. Cambridge University Press (1997)

21. Ben-Avraham, D.: Complete exact solution of diffusion-limited coalescence, A + A $\rightarrow$ A. Phys. Rev. Lett. 81, 4756 (1998)

22. Henkel, M., Hinrichsen, H.: Exact solution of a reaction-diffusion process with three-site interactions. J. Phys. A34, 1561-1568 (2001)

23. Mobilia, M., Bares, P.A.: Generalized empty-interval method applied to a class of one-dimensional stochastic models. Phys. Rev. E64, 066123 (2001)

24. Aghamohammadi, A., Khorrami, M.: Models solvable through the empty-interval method. Eur. Phys. J. B47, 583586 (2005)

25. Alimohammadi, M., Khorrami, M., Aghamohammadi: Exactly solvable models through the empty-interval method. Phys. Rev. E64, 056116 (2001)

26. Khorrami, M., Aghamohammadi, A., Alimohammadi, M.: Exactly solvable models through the empty interval method, for more-than-two-site interactions. J. Phys. A36, 345 (2003)

27. Aghamohammadi, A., Alimohammadi, M., Khorrami, M.: Exactly solvable models through the generalized empty interval method, for multi-species interactions. Eur. Phys. J. B31, 371 (2003)

28. Aghamohammadi, A., Khorrami, M.: Exactly solvable modeles through the generalized empty interval method: multi species and more-than-two-site interactions. Int. J. Modern Phys. B18, 2047 (2004)

29. Rozikov, U.A.: A contour method on Cayley trees. J. Stat. Phys. 130, 801-813 (2008) 